\title{
Home Hemodialysis Dose: Balancing Patient Needs and Preferences
}

\author{
Jose A. Diaz-Buxo ${ }^{a}$ Corinne E. Zeller-Knuth ${ }^{a}$ Kerry Anne Rambaran ${ }^{a}$ b \\ Rainer Himmele ${ }^{a}$ \\ ${ }^{a}$ Fresenius Medical Care North America, Waltham, Mass., and b Wingate University School of Pharmacy, \\ Wingate, N.C., USA
}

\section{Key Words}

Home hemodialysis · Hemodialysis prescription .

Dialysis dosing · Quality of life

\section{Abstract}

Background: The aim in defining the dose of HHD is to provide sufficient dialysis required to possibly 'normalize' all abnormalities associated with renal failure in order improve patient survival and quality of life. Much progress has been made in defining the dose required to accomplish this goal, but the evidence is still far from robust. The main limitations are incomplete understanding of uremic toxins, their relative importance in causing uremic symptoms, and our inability to comprehensively assess dry weight. Summary: This review provides guidance on realistic dosing of dialysis for the HHD patient based on the available evidence, where available, and alternative regimens that suit the individual's lifestyle and preferences. Key Messages: HHD can easily accommodate alternative, intensive HD prescriptions, including daily and nocturnal HD. HHD provides prescription flexibility to fulfill patient needs while taking their preferences into account.

(c) 2015 S. Karger AG, Basel

\section{Introduction}

Utilization of home hemodialysis (HHD) has been fueled in recent years by interest in more frequent or longer hemodialysis (HD) sessions along with the development of more patient-friendly equipment. In 2007, 2,706 (0.79\%) prevalent HD patients were utilizing home HD in the United States; in 2011 that number more than doubled to 5,535 (1.4\%) [1]. But, the percentage of home HD patients pales in comparison to those in the late 1960s and early 1970 s when around $40 \%$ of HD patients were receiving treatment at home. Among the many hurdles of HHD are concern for patient safety, lack of knowledge, and education by nurses and nephrologists about home therapies, the small number of facilities capable of training patients, and concerns by patients about technical aspects, social isolation, and family burden. Despite these obstacles, there has been resurgence in HHD due to better information on therapeutic choices, more flexibility, and control over their daily schedules, and the notion that HHD might improve patient outcomes and survival.

Dialysis dosing currently focuses on toxin removal, particularly that of urea. However, no standards have

\section{KARGER 125}

( 2015 S. Karger AG, Base

0253-5068/15/0393-0045\$39.50/0
Jose A. Diaz-Buxo, MD, FACP

309 East Morehead Street, Suite 285 Charlotte, NC 28202 (USA)

E-Mail jose.diaz-buxo@fmc-na.com 
been established for HHD beyond urea clearance. This review will emphasize the importance of a holistic and patient-centric prescription dictated by the analysis of the available evidence and patient preferences.

\section{Frequency and Time}

For patients on dialysis, conventional in-center HD (CHD) prescriptions consist of 3 weekly sessions of 3-4 hours each. In recent years, a number of observational cohort studies and the Frequent Hemodialysis Network (FHN) Trial [2,3] have investigated the role of intensified HD through increased frequency, increased duration of HD sessions, or both [4]. These intensified HD regimens include short daily HD (5-6 days/week for 1-2 hours), every-other day HD (3-4 hours every other day), daily or thrice weekly nocturnal HD (6-8 h/session), or extended HD ( $>4 \mathrm{~h}$ for 3 sessions/week). All of the alternative HD prescriptions, regardless of frequency and length, can be accommodated in the home setting.

There are risks and benefits to each HD regimen, including home use. Increasing overall HD treatment time has been shown to improve fluid volume and blood pressure control, require less use of phosphate binders, and lower mortality. However, these treatment regimens also have the potential to increase costs and can be perceived by patients to be less convenient and tolerable than CHD. Increasing the duration of $\mathrm{HD}$ sessions can improve clearance of middle molecules and larger solutes, and use lower ultrafiltration rates; but this approach can also lead to hypophosphatemia and more rapid loss of residual renal function [5]. Increasing frequency of HD can improve left ventricular hypertrophy; but can lead to vascular access complications.

\section{Dialysis Adequacy}

Historically, dialysis adequacy has been based upon urea kinetics, most often $\mathrm{Kt} / \mathrm{V}$, and was defined when small surface, low-flux dialyzers were used. The establishment of dialysis adequacy standards started with the $\mathrm{Na}-$ tional Cooperative Dialysis Study (NCDS). This study was the first trial to develop a quantifiable definition of dialysis treatment and they found thrice weekly HD was the pivotal point with respect to patient survival [6]. Next, in a mechanistic analysis of the NCDS, Gotch and Sargent defined Kt/V (clearance of urea $(\mathrm{K})$ multiplied by the time (t) of the dialysis treatment session, divided by the volume of distribution of urea in the body $(\mathrm{V})$ - which approximates the total body water volume) and concluded that fully adequate dialysis is achieved with a Kt/V of 1.0 [7]. Finally, the HEMO Study found no further benefit to achieving a single pool (sp) Kt/V greater than 1.2 [8]. Given this evidence, the National Kidney Foundation Dialysis Outcomes Quality Initiative (KDOQI) guidelines have since recommend a target spKt/V of 1.4 in order to achieve a minimum spKt/V of no less than 1.2 for $\mathrm{CHD}$ [9]. However, determining adequacy solely based on spKt/V can be misleading and lead to potential errors, especially in small or malnourished patients. This parameter quantifies the kinetic behavior of urea and is limited to urea kinetics without consideration of other components of the uremic syndrome and variability in modalities.

For alternative HD prescriptions, a different measure of adequacy must be used in order to accommodate the variable time and volume parameters. KDOQI recommends a minimal standard $\mathrm{Kt} / \mathrm{V}(\mathrm{stdKt} / \mathrm{V})$ target of 2.0 per week [9]. stdKt/V is derived from spKt/V and equilibrated $\mathrm{Kt} / \mathrm{V}$, and using stdKt/V as a measure of adequacy allows for the comparison of weekly urea clearance between HD regimens, including HHD [10]. However, we still have to consider other uremic toxins, fluid volume management, hemodynamic instability, clinical symptoms, and biochemical parameters, all of which have been associated with patient outcomes.

In addition to clearance of uremic toxins, dialysis directly affects a number of other parameters, including middle molecule clearance, bone mineral metabolism (BMM) markers, sodium balance, and fluid management; each of these is influenced differently by treatment frequency and duration of dialysis. The following section is a brief discussion of these parameters and how HHD could impact each.

As discussed above, urea has been the prototypical uremic marker and its removal is currently the standard for dialysis adequacy. Unfortunately, urea is not a typical uremic molecule, and its pattern of removal via dialysis is anything but representative [11]. Conventional HD removes urea quickly and efficiently, while removal of other toxins, that is, middle molecules, is less efficient. In fact, studies have demonstrated that prolonged and/or more frequent dialysis, or alternative modalities such as convective therapies, can better clear those larger toxins. Of the more than 50 uremic middle molecules [12], the most commonly measured is $\beta 2$-microglobin $(\beta 2 \mathrm{M})$, although, $\beta 2 \mathrm{M}$ is not necessarily representative of other middle molecules [11]. Several small clinical studies have looked at the effect of HD duration on the removal of $\beta 2 \mathrm{M}$ and
Diaz-Buxo/Zeller-Knuth/Rambaran/ Himmele 
found similar results: prolonged $\mathrm{HD}$, including nocturnal $\mathrm{HD}$, results in significantly better removal of $\beta 2$ microglobin than conventional HD; shorter, more frequent dialysis does not significantly impact $\beta 2 \mathrm{M}$ clearance [13].

BMM markers are also affected by dialysis frequency and length. While not considered uremic toxins, they do accumulate in kidney disease patients. Phosphate, calcium, and magnesium are small, water-soluble entities, but their removal rate is quite different. For example, phosphate clearance is complicated by the hydration shell around the phosphate ion and body compartmentalization. Thus, phosphate acts as a middle molecule during dialysis. Prolonged dialysis sessions (e.g., nocturnal HD) have been shown to significantly improve phosphate removal, even to the point that supplementation may be required [14].

Fluid gain and loss during and between HD sessions have a significant impact on hemodynamics. Underestimating dry weight or using a high ultrafiltration rate can result in underhydration, hypotension, and 'crash' during dialysis. The magnitude of inter- and intra-dialytic shifts is affected by the dialysis prescription. Acute and timeaveraged fluid overload is a key factor in the development of hypertension, edema, pulmonary congestion, and left ventricular hypertrophy in dialysis patients [15]. Both acute and time-averaged fluid levels are better managed by more frequent or prolonged HD [4].

Fluctuating sodium levels can result in vascular instability due to hypotension, which results in disequilibrium syndrome (fatigue, nausea, muscle cramps, and headache), frequently requiring saline infusions. More frequent HHD can improve sodium management and may better facilitate achievement of patient-specific goals. Consequently, prolonged or more frequent HHD can prevent significant inter- and intra-dialytic variation in sodium levels [16], which can, in turn, prevent the aforementioned complications.

$\mathrm{HD}$ also indirectly influences other parameters and outcomes including cardiovascular health and inflammation, nutrition, frequency of hospitalizations, quality of life, and survival. Moving dialysis into the home and allowing for more frequent or longer dialysis sessions can improve many of these secondary parameters.

Cardiovascular disease is disproportionate in the dialysis population and is closely associated with inflammation. One parameter of cardiovascular disease that is especially important in dialysis patients is blood pressure. The evidence for improved blood pressure control, which is tightly linked to hydration status, with intensified HD is robust [4]. Improvements in left ventricular mass (LVM) have also been found in numerous clinical studies with intensified HD [4]. Generally, improvements in BP and LVM have been attributed to the more physiological removal of fluid with higher frequency or longer dialysis sessions.

Regarding inflammation, Tomita et al. found a correlation between high levels of $\mathrm{C}$ reactive protein (CRP), an acute phase inflammatory marker, and intradialytic hypotension [17]. In a cross-sectional study comparing different HD regimens, daily and nocturnal HHD were associated with lower CRP; intradialytic hypotension was improved with daily HHD, and completely absent in nocturnal HHD patients [18]. Frequent HD schedules were associated with reduced levels of dialysis-induced cardiac injury (myocardial stunning) [18]. While increased exposure of the patient to the dialyzer membrane and dialysate with increased dialysis frequency or duration could contribute to increased microinflammation, intensified HHD might more effectively remove mediators of inflammation and oxidation $[4,19]$.

Balancing nutrition with dietary restrictions is challenging in ESRD patients. Nutrition in dialysis patients is routinely assessed using serum albumin as a marker for overall nutritional status since it has been known to associate tightly with malnutrition. Nutritional parameters have been examined in a number of studies on intensified $\mathrm{HD}$, both in-center and at home, but the data are inconsistent [20]. Most recently, the effect of thrice weekly, nocturnal hemodialysis on nutrition was examined in 55 conventional and 57 nocturnal patients [21]. After 12 months of prescribed treatment, patients on nocturnal HD had significantly improved serum albumin compared to conventional HD patients. Similar positive results were also found in two smaller studies on the effect of conversion from CHD to nocturnal HHD [20]. In contrast to these studies, the FHN Trial Daily and Nocturnal arms found no statistically significant differences between groups from baseline to 12 months in serum albumin measurements [2], although this parameter was underpowered.

Daily or nocturnal HHD can result in improved clearance of some uremic toxins over standard thrice weekly dialysis. Thus, it has been hypothesized that alternative HD regimens can lead to a reduction in the frequency of hospitalizations. A number of prospective and retrospective studies examined the effect of alternative HD regimens on hospitalizations, albeit with inconsistent results. Most recently, a large cohort study compared all-cause and cardiovascular-related hospitalization rates in $\mathrm{CHD}$ 
and daily HHD. There was no significant difference in all-cause hospitalization, but cardiovascular-related rates in HHD patients were lower, while infection-related cases were higher [22]. The FHN Daily and Nocturnal Trial concluded no clinically significant difference in hospitalizations between those receiving nocturnal HD and those receiving conventional $\mathrm{HD}$ [2]. A retrospective cohort that included 94 nocturnal HD patients, 34 SDHD and 1,370 CHD patients also reported no significant differences in hospitalizations between patients receiving NHD and CHD [23]. However, Bergman et al., in a cohort study that included $42 \mathrm{CHD}$ and $32 \mathrm{NHD}$ patients, reported improved hospitalization rates following conversion to NHD, while CHD hospitalization rates did not vary significantly for the duration of the study [24]. Longer duration RCTs with larger sample sizes including parallel arm comparators are needed to ascertain the definitive effects of frequent $\mathrm{HD}$, including $\mathrm{HHD}$, on hospitalization rates.

Numerous clinical trials and observational studies have included measures of quality of life when comparing CHD to intensified HD, both in-center and at home. However, drawing conclusions is complicated by the lack of a universal, objective standard for measuring QoL in dialysis patients. Although improvements in kidney-specific aspects of QoL have been reported in a number of clinical trials, data on overall QoL have been inconsistent [25]. With chronic conditions such as kidney disease, it is important to consider the long-term effects on QoL. Several observational studies with frequent or nocturnal HD patients have considered the long-term effects on QoL, one of which looked at 15 years of data [26], and found consistent improvements compared to CHD patients [4]. Generally, the QoL results in these studies support the notion that intensified HHD improves QoL. However future, well-powered, RCTs that employ objective QoL measures are needed in order to draw definitive conclusions.

For intensified HD regimens, there have been numerous observational studies on survival differences between HD prescriptions, but the only RCT has been the FHN Trial, which was underpowered. In matched cohort studies of both in-center and home HD patients comparing CHD to short daily HD $[27,28]$ and nocturnal HD [23, 29,30 ] survival was significantly improved with the intensified regimens. Two studies have specifically compared HHD with in-center HD and found improvements in survival independent of the total number of sessions per week [31, 32]. However, it is possible that selection bias played a role in influencing the results of these studies and further studies are needed.

\section{Patient Empowerment and Technology}

Dialysis patients must be able to make daily decisions regarding fluid intake, medications, diet, scheduling dialysis, and balancing their lives with their health needs. Sound education and good understanding of the dialytic prescription can improve patient compliance. Treatment, quality of life, and outcomes can improve with better education and patient empowerment [33]. Once a patient becomes an active participant in their treatment plans, the physician and patient can establish a variety of alternative HHD prescriptions to suit the patient's metabolic needs and personal lifestyle preferences. This could mean a patient is making the day-to-day decision at home on whether to do a short-daily, conventional, or long nocturnal dialysis session, as long as they are meeting the treatment goals prescribed by the physician. Using the technology available today [34], the HHD dose can be continuously customized accordingly in order to provide a much needed balance between improving health outcomes and quality of life. With the integration of remote monitoring and 'telemedicine' technologies the dialysis care team (patient, caregiver, physician, nurses, dieticians, and social workers) can be in continuous communication to ensure quality of care, compliance, and health status. Integrating remote monitoring in HHD can be achieved using telephone or Internet connection, whereby the patient and health care team receive real-time information.

\section{Conclusions}

The existing guidelines do not specifically address the home hemodialysis prescription, most likely due to the fact that there is no high-level evidence to justify such guidelines. Current dialysis dosing guidelines are evidence-based and represent the best of the available knowledge.

With the additional prescription flexibility that HHD offers, it is conceivable that the current standards could be exceeded. Newer, validated, and more efficacious methods are needed to assess adequacy that take into account the multitude variables affecting the health of ESRD patients. Perhaps adequacy should be addressed with a matrix of relevant parameters rather than toxin removal alone.

HHD can easily accommodate conventional as well as intensified HD prescriptions, be it more frequent or longer dialysis sessions, or both. In the home setting, patients can easily complete their dialysis sessions uninterrupted
48

Blood Purif 2015;39:45-49

DOI: $10.1159 / 000368944$
Diaz-Buxo/Zeller-Knuth/Rambaran/ Himmele 
and achieve their prescribed dialysis goals that can lead to better clinical parameters, health outcomes, and increased quality of life. HHD can be thought of as an individualized model that provides a unique opportunity to better educate the patients on their treatment needs and the role dialysis plays as their clinical condition changes. Furthermore, HHD can improve compliance with dialysis prescriptions as the patient becomes more familiar with the therapy regimen, thereby being able to achieve a balance between his or her needs and preferences.

\section{References}

1 US Renal Data System: USRDS 2013 Annual Data Report: Atlas of Chronic Kidney Disease and End-Stage Renal Disease in the United States, 2013.

2 Rocco MV, Lockridge RS, Beck GJ, et al: The effects of frequent nocturnal home hemodialysis: the Frequent Hemodialysis Network Nocturnal Trial. Kidney Int 2011;80:10801091.

-3 Chertow GM, Levin NW, Beck GJ, et al: Incenter hemodialysis six times per week versus three times per week. N Engl J Med 2010;363 2287-2300.

4 Diaz-Buxo JA, White SA, Himmele R: Frequent hemodialysis: a critical review. Semin Dial 2013;26:578-589.

5 Hakim RM, Saha S: Dialysis frequency versus dialysis time, that is the question. Kidney Int 2014;85:1024-1029.

6 Lowrie EG, Laird NM, Parker TF, et al: Effect of the hemodialysis prescription of patient morbidity: report from the National Cooperative Dialysis Study. N Engl J Med 1981;305 1176-1181.

7 Gotch FA, Sargent JA: A mechanistic analysis of the National Cooperative Dialysis Study (NCDS). Kidney Int 1985;28:526-534.

8 Eknoyan G, Beck GJ, Cheung AK, et al: Effect of dialysis dose and membrane flux in maintenance hemodialysis. N Engl J Med 2002; 347:2010-2019.

-9 Gilmore J: KDOQI clinical practice guidelines and clinical practice recommendations 2006 updates. Nephrol Nurs J 2006;33:487-488.

10 Gotch FA: The current place of urea kinetic modelling with respect to different dialysis modalities. Nephrol Dial Transplant 1998; 13(suppl 6):10-14.

-11 Fagugli RM, De Smet R, Buoncristiani U, et al: Behavior of non-protein-bound and proteinbound uremic solutes during daily hemodialysis. Am J Kidney Dis 2002;40:339-347.

12 Duranton F, Cohen G, De Smet R, et al: Nor$\mathrm{mal}$ and pathologic concentrations of uremic toxins. J Am Soc Nephrol 2012;23:1258-1270.
13 Cornelis T, van der Sande FM, Eloot S, et al: Acute hemodynamic response and uremic toxin removal in conventional and extended hemodialysis and hemodiafiltration: a randomized crossover study. Am J Kidney Dis 2014;62:247-256.

14 Daugirdas JT, Chertow GM, Larive B, et al: Effects of frequent hemodialysis on measures of CKD mineral and bone disorder. J Am Soc Nephrol 2012;23:727-738.

15 Velasco N, Chamney P, Wabel P, et al: Optimal fluid control can normalize cardiovascular risk markers and limit left ventricular hypertrophy in thrice weekly dialysis patients. Hemodial Int 2012;16:465-472.

16 Santos SF, Peixoto AJ: Sodium balance in maintenance hemodialysis. Semin Dial 2010 23:549-555.

17 Tomita M, Malhotra D, Dheenan S, et al: A potential role for immune activation in hemodialysis hypotension. Ren Fail 2001;23:637-649.

18 Jefferies HJ, Virk B, Schiller B, et al: Frequent hemodialysis schedules are associated with reduced levels of dialysis-induced cardiac injury (myocardial stunning). Clin J Am Soc Nephrol 2011;6:1326-1332.

19 Yuen D, Richardson RM, Fenton SS, et al: Quotidian nocturnal hemodialysis improves cytokine profile and enhances erythropoietin responsiveness. ASAIO J 2005;51:236-241.

20 Rocco MV: Does more frequent hemodialysis provide dietary freedom? J Ren Nutr 2013;23: 259-262.

21 Demirci C, Ozkahya M, Demirci MS, et al: Effects of three times weekly eight-hour nocturnal hemodialysis on volume and nutritional status. Am J Nephrol 2013;37:559-567.

-22 Weinhandl ED, Nieman KM, Gilbertson DT, et al: Hospitalization in daily home hemodialysis and matched thrice-weekly in-center hemodialysis patients. Am J Kidney Dis 2014, DOI: 10.1053/j.ajkd.2014.06.015, Epub ahead of print.

23 Johansen KL, Zhang R, Huang Y, et al: Survival and hospitalization among patients using nocturnal and short daily compared to conventional hemodialysis: a USRDS study. Kidney Int 2009;76:984-990.
24 Bergman A, Fenton SS, Richardson RM, et al Reduction in cardiovascular related hospitalization with nocturnal home hemodialysis. Clin Nephrol 2008;69:33-39.

25 Tennankore K, Nadeau-Fredette AC, Chan CT: Intensified home hemodialysis: clinical benefits, risks and target populations. Nephrol Dial Transplant 2014;29:1342-1349.

26 Buoncristiani U: Fifteen years of clinical experience with daily haemodialysis. Nephrol Dial Transplant 1998;13(suppl 6):148-151.

27 Blagg CR, Kjellstrand CM, Ting GO, et al: Comparison of survival between short-daily hemodialysis and conventional hemodialysis using the standardized mortality ratio. Hemodial Int 2006; 10:371-374.

28 Weinhandl ED, Liu J, Gilbertson DT, et al: Survival in daily home hemodialysis and matched thrice-weekly in-center hemodialysis patients. J Am Soc Nephrol 2012;23:895904

29 Nesrallah GE, Lindsay RM, Cuerden MS, et al Intensive hemodialysis associates with improved survival compared with conventional hemodialysis. J Am Soc Nephrol 2012;23: 696-705.

30 Lockridge RS, Kjellstrand CM: Nightly home hemodialysis: outcome and factors associated with survival. Hemodial Int 2011;15:211218.

31 Marshall MR, Hawley CM, Kerr PG, et al: Home hemodialysis and mortality risk in Australian and New Zealand populations. Am J Kidney Dis 2011;58:782-793.

32 Woods JD, Port FK, Stannard D, et al: Comparison of mortality with home hemodialysis and center hemodialysis: a national study. Kidney Int 1996;49:1464-1470.

33 White RD: Patient empowerment and optimal glycemic control. Curr Med Res Opin 2012;28:979-989.

34 Cafazzo JA, Leonard K, Easty AC, et al: Patient perceptions of remote monitoring for nocturnal home hemodialysis. Hemodial Int 2010;14:471-477. 